\title{
Niveles de fósforo y sus efectos sobre el crecimiento y consumo en alpacas hembras en los Andes peruanos
}

\author{
Phosphorus levels and their effects on growth and intake in female alpacas in the \\ Peruvian Andes
}

\author{
Carlos Quispe E. ${ }^{1,4}$, Edith Ancco G. ${ }^{1}$, Robert Van Saun ${ }^{2}$, Carlos Gómez B. ${ }^{3}$
}

\section{Resumien}

\begin{abstract}
El estudio tuvo como objetivo conocer los niveles de fósforo y sus efectos sobre el consumo de alimento y peso vivo en alpacas hembras durante la temporada de reproducción (enero-febrero) y el destete (agosto-septiembre). En la primera etapa se determinaron los niveles séricos de fósforo en 200 alpacas de 11-13 meses de edad, ubicadas en cuatro regiones alpaqueras del Perú. Los niveles de P sérico fueron de $8.25 \pm 1.63,5.25 \pm 1.46$, $6.42 \pm 1.84$ y $4.8 \pm 1.61 \mathrm{mg} / \mathrm{dl}$ para los animales de las regiones de Pasco, Junín, Cuzco y Puno, respectivamente, habiendo diferencias significativas $(\mathrm{p}<0.05)$ entre regiones. Una región se encontró por debajo del límite de referencia $(5-11.5 \mathrm{mg} / \mathrm{dl})$ y otra cerca del límite inferior, pudiendo considerarse como deficientes en P. En la segunda etapa se realizó un experimento controlado con tres concentraciones de P en la dieta: $0.16 \%$ (T1), $0.25 \%$ (T2) y $0.34 \%$ (T3), con 16 alpacas hembras destetadas (7-8 meses, $25.25 \pm 1.62 \mathrm{~kg}$ ) por grupo. Los animales recibieron alimentación ad libitum y estuvieron alojados en corrales individuales durante siete meses. El consumo de materia seca fue de $0.717 \pm 0.82$, $0.826 \pm 0.82$ y $0.967 \pm 0.49 \mathrm{~kg} / \mathrm{animal} /$ día, mientras que, como porcentaje del peso vivo fue $2.07,2.24$ y 2.60 para los tratamientos T1, T2 y T3, respectivamente. En ambos casos hubo diferencias significativas entre tratamientos $(\mathrm{p}<0.05)$. Hubo una reducción significativa en la concentración de P en T1 $(5.02 \pm 1.11 \mathrm{mg} / \mathrm{dl})$ comparado con T2 $(11.07 \pm 1.79 \mathrm{mg} / \mathrm{dl})$ y T3 $(11.45 \pm 1.14 \mathrm{mg} / \mathrm{dl})$. Es probable que el bajo fósforo dietario en T1 pudo haber afectado el consumo y la ganancia de peso en alpacas en crecimiento.
\end{abstract}

Palabras clave: fósforo; alpacas; crecimiento; consumo; destete

\footnotetext{
${ }^{1}$ Programa Doctoral en Ciencia Animal, Escuela de Pos Grado, Universidad Nacional Agraria La Molina, Lima, Perú

${ }^{2}$ Departamento de Veterinaria y Ciencias Biomédicas, Pennsylvania State University, USA

${ }^{3}$ Departamento de Nutrición Animal, Facultad de Zootecnia, Universidad Nacional Agraria La Molina, Lima, Perú

${ }^{4}$ E-mail: cquispe.vet@gmail.com
}

Recibido: 5 de octubre de 2018

Aceptado para publicación: 10 de abril de abril de 2019 
The aim of this study was to know the levels of phosphorus and its effects on feed intake and body weight in female alpacas during the breeding season (January-February) and weaning (August-September). In the first phase, serum phosphorus levels were determined in 200 11-13-month-old alpacas, located in four alpaca regions of Peru. P levels were $8.25 \pm 1.63,5.25 \pm 1.46,6.42 \pm 1.84$ and $4.8 \pm 1.61 \mathrm{mg} / \mathrm{dl}$ for the regions of Pasco, Junín, Cuzco and Puno, respectively, with significant differences $(\mathrm{p}<0.05)$ between regions. $P$ values in one region were below the reference limit $(5-11.5 \mathrm{mg} / \mathrm{dl})$ and the another near the lower limit, which could be considered as deficient in P. In the second phase, a controlled experiment was carried out with three concentrations of $\mathrm{P}$ in the diet: $0.16 \%$ (T1), $0.25 \%$ (T2) and $0.34 \%$ (T3), using 16 weaned female alpacas per group (7-8 months, $25.25 \pm 1.62 \mathrm{~kg}$ ). The animals received the feeds ad libitum and were housed in individual pens for seven months. Dry matter intake was $0.717 \pm 0.82,0.826 \pm 0.82$ and $0.967 \pm 0.49$ $\mathrm{kg} / \mathrm{animal} / \mathrm{day}$ and as a percentage of live weight was $2.07,2.24$ and 2.60 for treatments $\mathrm{T} 1, \mathrm{~T} 2$ and $\mathrm{T} 3$, respectively. In both cases there were significant differences between treatments $(\mathrm{p}<0.05)$. There was a significant reduction in the concentration of P in T1 $(5.02 \pm 1.11 \mathrm{mg} / \mathrm{dl})$ compared to T2 $(11.07 \pm 1.79 \mathrm{mg} / \mathrm{dl})$ and T3 $(11.45 \pm 1.14 \mathrm{mg} / \mathrm{dl})$. It is likely that low dietary phosphorus in T1 may have affected consumption and weight gain in growing alpacas.

Key words: phosphorus; alpacas; growth; intake; weaning

\section{INTRODUCCIÓN}

Alrededor del $90 \%$ de las alpacas están en manos de pequeños productores y comunidades campesinas, siendo su fuente económica más importante (Ramírez, 1990; More et al., 2011). La oportunidad de encontrar pastizales sobre los $3800 \mathrm{msnm}$ que ofrezcan una dieta con adecuadas concentraciones de nutrientes que se ajusten a los requerimientos de los camélidos es muy baja. Las deficiencias de proteína, energía y algunas vitaminas y minerales son severas, coincidiendo con etapas críticas en la vida productiva de las alpacas, como son el destete y el último tercio de gestación (San Martín, 1996).

El fósforo es uno de los minerales deficientes en los pastizales (San Martin y Bryant, 1989; Flores y Oscanoa, 1992; Waldridge y Pugh, 1997), cuya relación con funciones productivas y reproductivas ha sido descrita por varios autores y en varias especies. Una de las alteraciones de primer orden es el retraso en el inicio de la pubertad, encontrándose que más del $80 \%$ de las alpacas destetadas no llegan a alcanzar el peso adecuado o mínimo requerido para ser servidas $(>35 \mathrm{~kg})$ al año de edad en la estación reproductiva de enero a marzo (Huanca et al., 2015; San Martín, 2015). Además, el destete coincide con la época seca donde los requerimientos nutricionales son difícilmente cubiertos y esto trae como consecuencia que los animales no alcancen pesos adecuados, retrasando la pubertad y la función ovárica; de allí que los criadores se ven obligados a empadrar a los dos años a estos animales, asumiendo las consecuencias económicas que esto implica.

El fósforo cumple con el mantenimiento de la presión osmótica y el equilibrio ácido-básico, la formación de fosfolípidos y, en consecuencia, en el transporte de ácidos grasos y en la formación de aminoácidos y proteínas (Vitti et al., 2010). Van Saun (2006) menciona que el metabolismo del fósforo en rumiantes y especialmente en camélidos es único, donde el fósforo de la sangre es reciclado al rumen a través de la saliva. 
Cuadro 1. Insumos y proporciones usadas en las dietas experimentales con tres niveles de fósforo (segunda fase del estudio)

\begin{tabular}{lccc}
\hline \multirow{2}{*}{ Insumo } & \multicolumn{3}{c}{ Proporciones en la dieta (\%) } \\
\cline { 2 - 4 } & $0.16 \% \mathrm{P}$ & $0.25 \% \mathrm{P}$ & $0.34 \% \mathrm{P}$ \\
\hline Heno de avena & 68.7 & 68.1 & 67.7 \\
Heno de alfalfa & 24.9 & 25.0 & 24.9 \\
Melaza & 4 & 4 & 4 \\
Urea & 1.9 & 1.9 & 1.9 \\
Sal común & 0.3 & 0.3 & 0.3 \\
Suplemento vitamínico & 0.2 & 0.2 & 0.2 \\
Fosfato dicálcico & ----- & 0.5 & 1.0 \\
\hline Total & 100 & 100 & 100 \\
\hline
\end{tabular}

La bioquímica sanguínea es la valoración de la concentración de metabolitos en la sangre, representa un análisis integrado de la adecuación del suministro de nutrientes con relación a su utilización (Marín et al., 2016). Se han reportado estudios sobre parámetros bioquímicos en camélidos sudamericanos, tanto en buena condición de salud como en enfermedad (Anderson, 2002); sin embargo, existe escasa información sobre la bioquímica sanguínea de la alpaca en diferentes etapas de su vida productiva, sobre todo en etapas críticas de su producción, como es el destete. Con el fin de implementar un esquema de manejo nutricional adecuado con relación al fósforo, el presente estudio tuvo como objetivo conocer los niveles de fósforo en alpacas hembra posdestete y en dietas en cuatro regiones del Perú, además de determinar el efecto del fósforo en la dieta y sus posibles implicancias sobre el consumo y el crecimiento en la alpaca.

\section{Materiales y Métodos}

El estudio tuvo dos fases. En la primera etapa se determinaron los niveles sanguíneos de fósforo en 200 alpacas hembras de 11-13 meses de edad durante la estación repro- ductiva (febrero) en las cuatro regiones alpaqueras de mayor importancia (Puno, Junín, Pasco y Cusco) del Perú. Además, se determinaron las concentraciones de fósforo en las pasturas consumidas por 40 alpacas (10 por región). Las pasturas fueron colectadas mediante la técnica de simulación manual (Austin et al., 1983). Cada pastura estuvo compuesta por 30 estaciones alimentarias, las que se definen como el semicírculo en frente del animal dentro del cual el animal cosecha el forraje cada vez que se detiene a comer (Flores, 1983). La composición botánica de cada dieta estuvo compuesta por grupos funcionales como gramíneas altas y bajas, ciperáceas, juncáceas, rosáceas y hierbas. Solo se determinaron las concentraciones de fósforo.

La segunda fase del estudio se llevó a cabo en la Cooperativa Comunal «San Pedro de Racco», ubicada en el distrito de Simón Bolívar en la región de Cerro de Pasco, Perú, a una altitud de $4318 \mathrm{msnm}$ y con temperatura promedio de $8^{\circ} \mathrm{C}$ entre setiembre y marzo. Se utilizaron 48 alpacas posdestete con una edad promedio de 8 meses y un peso vivo promedio de $25.2 \pm 1.6 \mathrm{~kg}$. Los animales fueron alojados en corrales individuales durante siete meses, tuvieron alimento y agua ad libitum y fueron 
Cuadro 2. Composición química de las dietas experimentales conteniendo tres niveles de fósforo (segunda fase del estudio)

\begin{tabular}{lccc}
\hline \multirow{2}{*}{ Componente } & \multicolumn{3}{c}{ Niveles de fósforo } \\
\cline { 2 - 4 } & $0.16 \% \mathrm{P}$ & $0.25 \% \mathrm{P}$ & $0.34 \% \mathrm{P}$ \\
\hline Materia Seca (\%) & 85.10 & 85.10 & 85.15 \\
Proteína (\%) & 12.11 & 12.11 & 12.07 \\
EM (Mcal $/ \mathrm{kg})$ & 1.88 & 1.87 & 1.86 \\
NDT (\%) & 58.67 & 57.43 & 59.23 \\
P (\%)** & 0.166 & 0.252 & 0.347 \\
\hline
\end{tabular}

distribuidos en un experimento controlado con tres concentraciones de fósforo en la dieta: $0.16 \%$ (T1), $0.25 \%$ (T2) y $0.34 \%$ (T3) (Cuadros 1 y 2). Los niveles de fósforo en las dietas experimentales fueron alcanzados mediante fósforo suplementario en una dieta base en el T2 y T3. El periodo de acostumbramiento a la dieta fue 20 días. El peso vivo fue determinado con una balanza electrónica de plataforma cada 30 días. El consumo fue considerado como la diferencia entre el peso del alimento ofrecido y el peso del alimento residual por día.

Las muestras de sangre $(3-5 \mathrm{ml})$ fueron recogidas en tubos tipo vacutainer de la vena yugular a las 06:00 y con los animales en ayunas; luego fueron almacenadas en cajas herméticas a $8{ }^{\circ} \mathrm{C}$ hasta su traslado al laboratorio. Se siguieron los protocolos de asepsia al momento de colectar la muestra cuidando la integridad del animal. Las muestras colectadas fueron centrifugadas a 1077 $\mathrm{x} g$ por 15 minutos y el suero sanguíneo resultante fue colocado en viales y congelado a $-20{ }^{\circ} \mathrm{C}$. La determinación de fósforo $(\mathrm{P})$ se realizó por espectrofotometría UV, mediante un kit comercial Valtek ${ }^{\circledR}$ (fósforo UV molibdato). La mayoría de los métodos para la determinación de fósforo inorgánico se basan en la formación de fosfomolibdato de amonio y en su posterior reducción a azul de molibdeno (Wang et al., 1983). La for- mación del complejo fosfomolíbdico no reducido se mide a $340 \mathrm{~nm}$, y la absorbancia obtenida es directamente proporcional a la concentración de fósforo inorgánico presente en la muestra.

Los datos fueron analizados mediante estadística descriptiva. Se hizo una prueba de normalidad para determinar la distribución normal de los datos de fósforo. Se realizó un análisis de varianza para determinar las diferencias entre tratamientos y una prueba de contingencia para evaluar las diferencias entre regiones. El nivel de significancia fue del $95 \%$. El paquete estadístico utilizado fue el SAS v. 9.0 y SPSS v. 15.0.

\section{Resultados}

En el Cuadro 3 se presentan las concentraciones de fósforo en las pasturas consumidas por las alpacas. Los valores más bajos se observan en la región Junín, seguido por Puno y Cusco, encontrándose las tres regiones por debajo del rango referencial propuesto por Van Saun y Herdt (2014). Con relación a los valores en suero, se encontraron diferencias significativas $(\mathrm{p}<0.05)$ entre las cuatro regiones (Cuadro 4), siendo deficientes los encontrados en la región Puno al ser comparados con los datos referenciales de Van Saun y Herdt (2014). 
Cuadro 3. Concentraciones de fósforo en las pasturas (\%) de 10 alpacas púberes por región del Perú

\begin{tabular}{lcc}
\hline Región & $\begin{array}{c}\text { Fósforo } \\
(\%)\end{array}$ & $\begin{array}{c}\text { Rango de } \\
\text { referencia } \\
(\%)\end{array}$ \\
\hline Pasco & 0.24 & \\
Junín & 0.10 & $0.2-0.4$ \\
Puno & 0.13 & \\
Cuzco & 0.13 & \\
\hline
\end{tabular}

${ }^{1}$ Van Saun y Herdt (2014)

Cuadro 4. Concentraciones de fósforo sanguíneo $(\mathrm{mg} / \mathrm{dl})$ en 40 alpacas púberes por región del Perú

\begin{tabular}{ccc}
\hline Región & $\begin{array}{c}\text { Fósforo } \\
(\mathrm{mg} / \mathrm{dl})\end{array}$ & $\begin{array}{c}\text { Rango de } \\
\text { referencia } \\
(\%)\end{array}$ \\
\hline Pasco & $\begin{array}{c}\text { 8.25 } \\
\text { Junín }\end{array}$ & $5.25 \pm$ \\
& $1.46^{\mathrm{b}}$ \\
& $4.80 \pm$ & $5.0-11.5$ \\
Puno & $1.61^{\mathrm{c}}$ \\
Cuzco & $6.42 \pm 1.84^{\mathrm{b}}$ \\
\hline a,b,c Superíndices diferentes dentro de \\
columnas indican diferencias entre \\
regiones (p<0.05)
\end{tabular}

En la segunda parte del estudio, los pesos de las alpacas de $\mathrm{T} 1$ al final del séptimo mes del estudio fueron estadísticamente diferentes a los pesos finales en T2 y $\mathrm{T} 3$ $(p<0.05)$, no habiendo diferencias entre estos dos últimos (Cuadro 5). El consumo de materia seca expresado en $\mathrm{kg} /$ día fue estadísticamente diferente entre los tres tratamientos (Cuadro 6); $\mathrm{p}<0.05$ ). Así mismo, hubo una apreciable reducción en la con- centración de fósforo en suero sanguíneo en las alpacas de T1 $(5.02 \pm 1.11 \mathrm{mg} / \mathrm{dl})$ comparado con las de $\mathrm{T} 2(11.07 \pm 1.79 \mathrm{mg} / \mathrm{dl})$ y de T3 (11.45 $\pm 1.14 \mathrm{mg} / \mathrm{dl}$ ) (Cuadro 7; $<<0.05)$. Las concentraciones de $\mathrm{P}$ en $\mathrm{T} 1$ podrían considerarse como deficiente si se comparan con los valores referenciales propuestos por Van Saun y Herdt (2014).

\section{Discusión}

Un aspecto interesante resultó al comparar los valores obtenidos con respecto a las concentraciones de fósforo en la dieta con el rango de valores esperados reportados por Van Saun y Herdt (2014), quienes señalan un rango de $5-11.5 \mathrm{mg} / \mathrm{dl}$ como valores normales. En el presente estudio, se observó que los animales de Pasco y Cusco se encontraron dentro del rango, los de Junín estaban en el límite inferior y los de Puno por debajo del rango. Esto puede estar relacionado a las deficiencias de $\mathrm{P}$ en los pastizales altoandinos (Flores y Oscanoa, 1992; Waldridge y Pugh, 1997), lo cual podría estar afectando algunos parámetros productivos, de consumo y crecimiento de los animales.

Las concentraciones de fósforo en suero de alpacas durante la estación reproductiva en dos de las cuatro regiones (una debajo del límite mencionado y otra en el límite) pueden considerarse como deficientes en fósforo. Esto también puede estar relacionado con las deficiencias de este mineral en los pastizales altoandinos reportados a pesar de que la evaluación fue realizada en época húmeda donde la oferta alimenticia se ve incrementada; sin embargo, tal como se observa en el Cuadro 3, las dietas de alpacas en esta época son deficientes en fósforo en la mayoría de las regiones.

En la segunda parte del estudio es probable que el bajo fósforo dietario en $\mathrm{T} 1$ pudo haber tenido influencia sobre el consumo y la ganancia de peso en alpacas en crecimiento. Las concentraciones de fósforo en T2 y T3 
Cuadro 5. Peso vivo inicial $(\mathrm{kg})$ en alpacas hembra posdestete (septiembre) y pesos finales (febrero) en alpacas púberes de cuatro regiones del Perú con dietas conteniendo tres niveles de fósforo

\begin{tabular}{lccc}
\hline Tratamientos & $\mathrm{n}$ & Peso inicial & Peso final \\
\hline T1 (Bajo fósforo) & 16 & $25.22 \pm 1.09^{\mathrm{a}}$ & $34.58 \pm 1.56^{\mathrm{b}}$ \\
T2 (Medio fósforo) & 16 & $25.19 \pm 0.98^{\mathrm{a}}$ & $36.8 \pm 1.03^{\mathrm{a}}$ \\
T3 (Alto fósforo) & 16 & $25.33 \pm 1.82^{\mathrm{a}}$ & $37.1 \pm 0.99^{\mathrm{a}}$ \\
\hline
\end{tabular}

$a, b$ Letras diferentes dentro de columnas indican diferencias entre tratamientos $(p<0.05)$

Cuadro 6. Consumo de materia seca $(\mathrm{kg} / \mathrm{d})$ y su relación con el peso vivo a la mitad (noviembre) y final del estudio (febrero) en alpacas púberes de cuatro regiones del Perú consumiendo dietas con tres niveles de fósforo

\begin{tabular}{lccccc}
\hline \multirow{2}{*}{ Tratamientos } & \multirow{2}{*}{$\mathrm{N}^{\circ}$} & \multicolumn{2}{c}{ Mitad del estudio } & \multicolumn{2}{c}{ Final del estudio } \\
\cline { 3 - 6 } & & $\mathrm{kg} / \mathrm{d} \mathrm{MS}$ & $\% \mathrm{BW}$ & $\mathrm{kg} / \mathrm{d} \mathrm{DM}$ & $\% \mathrm{BW}$ \\
\hline T1 (Bajo Fósforo) & 16 & $0.458 \pm 0.16^{\mathrm{c}}$ & 1.52 & $0.717 \pm 0.82^{\mathrm{c}}$ & 2.07 \\
T2 (Medio Fósforo) & 16 & $0.562 \pm 0.78^{\mathrm{b}}$ & 1.73 & $0.826 \pm 0.82^{\mathrm{b}}$ & 2.24 \\
T3 (Alto Fósforo) & 16 & $0.652 \pm 0.53^{\mathrm{a}}$ & 1.97 & $0.967 \pm 0.49^{\mathrm{a}}$ & 2.60 \\
\hline
\end{tabular}

$a, b, c$ Letras diferentes dentro de columnas indican diferencias entre tratamientos $(p<0.05)$

Cuadro 7. Concentraciones de fósforo en suero $(\mathrm{mg} / \mathrm{dl})$ al inicio y al final del estudio en alpacas de cuatro regiones del Perú consumiendo dietas conteniendo tres niveles de fósforo

\begin{tabular}{lccc}
\hline Tratamientos & $\mathrm{N}^{\circ}$ & $\begin{array}{c}\text { Concentraciones } \\
\text { iniciales }\end{array}$ & $\begin{array}{c}\text { Concentraciones } \\
\text { finales }\end{array}$ \\
\hline T1 (Bajo fósforo) & 16 & $6.44 \pm 1.32^{\mathrm{a}}$ & $5.02 \pm 1.11^{\mathrm{a}}$ \\
T2 (Medio fósforo) & 16 & $5.87 \pm 1.18^{\mathrm{a}}$ & $11.07 \pm 1.79^{\mathrm{b}}$ \\
T3 (Alto fósforo) & 16 & $6.15 \pm 1.22^{\mathrm{a}}$ & $11.45 \pm 1.14^{\mathrm{b}}$ \\
\hline
\end{tabular}

${ }^{a, b}$ Letras diferentes dentro de columnas indican diferencias entre tratamientos $(p<0.05)$ 
afectaron los parámetros bajo medición. El efecto del menor consumo en T1 puede haber influido en el aumento de peso. En ambos casos hubo diferencias significativas entre tratamientos $(\mathrm{p}<0.05)$. La reducción del consumo en T1 pudo haber afectado el peso vivo final. El bajo fósforo en la dieta probablemente afectó la eficiencia bacteriana en el compartimento $1(\mathrm{C} 1)$ de las alpacas.

\section{Conclusiones}

- Los resultados demuestran que las dietas de alpacas en crecimiento no ofrecen las cantidades adecuadas de fósforo y están en el límite o por debajo de los rangos referenciales.

- Se encontró un efecto del fósforo dietario sobre el consumo y la ganancia de peso. Una dieta con $0.16 \%$ evidencia un descenso marcado de los parámetros medidos, mientras que, un punto intermedio entre 0.25 y $0.34 \%$ puede considerarse como alternativa de requerimiento basado en la respuesta biológica.

\section{Literatura Citada}

1. Anderson D. 2002. Liver disease in camelids. USA: Ohio State University. [Internet]. Disponible en: https:// www.rmla.com $/ \mathrm{html} /$-LiverDisease.htm

2. Austin D, Urness P, Fierro L. 1983. Spring livestock grazing affect crested wheatgrass regrowth and winter use by mule deer. J Range Manage 36: 589-593.

3. Flores ER, Oscanoa L. 1992. Avances en la ecología, alpacas y llamas en el ecosistema de puna seca. Boletín Técnico 3: 2-11.

4. Flores E. 1983. Applying the concept of feeding stations to the behavior of cattle grazing variable amounts of available forage. MSc Thesis. Logan, Utah: Utah State University. 58 p
5. Huanca W, Cordero A, Huanca T. 2015. Relación entre la nutrición y el comportamiento reproductivo en los camélidos sudamericanos. En: VII Congreso Mundial de Camélidos Sudamericanos. Puno, Perú.

6. Marín R, Medina O, Corregidor P. 2016. Valores de calcio, fósforo, magnesio y proteínas en suero de llamas (Lama glama). En: XXI Reunión Científico Técnica de la Asociación Argentina de Veterinarios de Laboratorios de Diagnóstico. Jujuy, Argentina.

7. More J, Manchego A, Sandoval N, Ramirez M, Rivera H. 2011. Detección genómica y expresión de péptidos antimicrobianos (á- y â- defensinas) en mucosa intestinal de crías de alpaca (Vicugna pacos). Rev Inv Vet Perú 22: 324-335. doi: 10.15381/ rivep.v22i4.332

8. Ramírez A. 1990. Colibacilosis entérica en crías de Alpacas. En: Avances sobre investigación en salud animal de camélidos sudamericanos. UNMSM. Bol. Div. N. ${ }^{\circ} 23.64 \mathrm{p}$.

9. San Martín F. 1996. Nutrición de camélidos sudamericanos y su relación con la reproducción. Rev Argentina Prod Anim 16: 305-312.

10. San Martín F. 2015. Adaptación nutricional y metabólica de los camélidos sudamericanos. En: VII Congreso Mundial de Camélidos Sudamericanos. Puno, Perú.

11. San Martin F, Bryant FC 1989. Nutrition of domesticated South American llamas and alpacas. Small Ruminant Res 2: 191-216. doi: 10.1016/ 0921-4488(89)90001-1

12. Van Saun R, Herdt T. 2014. Nutritional assessment. In: Llama y alpaca care. Medicine, surgery, reproduction, nutrition, and herd health. Saunders. p 100-123.

13. Van Saun RJ. 2006. Nutrient requirements of South American camelids: a factorial approach. Small Ruminant Res 61: 165-186. doi: 10.1016/ j.smallrumres.2005.07.006 
14. Vitti D, Da Silva J, Louvandini H. 2010. Phosphorus and calcium utilization in ruminants using isotope dilution technique. In: Phosphorus and calcium utilization and requirements in farm animals. CAB International. p 45-67.
15. Waldridge BM, Pugh DG 1997. Managing trace mineral deficiencies in South American camelids. Vet Med 92: 744-750.

16. Wang J, Chen CC, Osaki S. 1983. Optimization of the phosphorus-UV reagent. Clin Chem J 29: 1255. [Abstract]. 